\title{
Controle de Ipomoea nil utilizando ponta centrífuga de pulverização em diferentes volumes de aplicação com e sem adjuvante ${ }^{1}$
}

\section{Control of Ipomoea nil using tip centrifugal machine of spraying in different volumes of the application with and without adjuvant}

\author{
Bruno Flávio Figueiredo Barbosa ${ }^{2 *}$ Marcelo da Costa Ferreira ${ }^{2}$ José Luiz da Silva Fabio Alexandre \\ Cavichioli $^{3}$ Rafael Scabello Bertonha ${ }^{3}$ Anselmo Augusto de Paiva Custódio ${ }^{4}$
}

Resumo - A incorporação de ferramentas relacionadas à tecnologia de aplicação de agrotóxicos torna-se inevitável para uma agricultura sustentável. Foi testada a eficiência no controle de Ipomoea nil, em pós-emergência inicial com diuron+hexazinone, em dois volumes de aplicação (20 L ha ${ }^{-1}$ e $40 \mathrm{~L} \mathrm{ha}^{-1}$ ), com ou sem a adição de adjuvantes, utilizando-se pontas de pulverização rotativo e hidráulico, comparativamente. Foram realizados outros dois ensaios para comparar a distribuição volumétrica e o diâmetro das gotas produzidas pelas pontas hidráulica e rotativa. $\mathrm{O}$ percentual de necrose na parte aérea de I. nil aumentou progressivamente, nos dois volumes, independentemente da utilização de adjuvante. A ponta rotativa não diferiu da ponta hidráulica quanto à eficácia. $\mathrm{O}$ uso da ponta rotativa diminuiu o coeficiente de uniformidade (COEF) em relação à ponta hidráulica, indicando produção de gotas com maior uniformidade. A distribuição das gotas produzidas pela ponta rotativa proporcionou padrão de distribuição desuniforme em relação à ponta testemunha (TT 110.01 a 40 L.ha $^{-1}$ ), devido ao menor volume de aplicação utilizado. O espaçamento entre pontas foi maior com o uso de óleo vegetal para o volume de $20 \mathrm{~L}$ $\mathrm{ha}^{-1}$. A associação dos adjuvantes à diuron + hexazinone proporcionou aumento do diâmetro médio das gotas, com semelhante eficiência entre os dois volumes testados. $\mathrm{O}$ menor volume de aplicação $\left(20 \mathrm{~L} \mathrm{ha}^{-1}\right)$ de diuron+hexazinone pode ser utilizado no controle de $I$. nil, pois proporcionou eficácia semelhante ao volume maior. A utilização dos adjuvantes organo siliconado $(0,025 \% \mathrm{v} / \mathrm{v})$ e óleo vegetal $(1,0 \% \mathrm{v} / \mathrm{v})$, pode ser recomendada em sistema de pulverização com bico rotativo, pois permite aumento do diâmetro médio das gotas e diminuição do risco de deriva, possibilitando a utilização do menor volume de aplicação $\left(20 \mathrm{~L} \mathrm{ha}^{-1}\right)$, por ter aumentando o potencial de sobreposição das gotas sobre o alvo.

\footnotetext{
${ }^{1}$ Recebido para publicação em 16/06/2011 e aceito em 10/11/2011.

Trabalho realizado com bolsa de estudo financiada pela CAPES e CNPq

${ }^{2}$ Departamento de Fitossanidade, Universidade Estadual Paulista (UNESP), Via de Acesso Professor Paulo Donato Castellane, s/nº, Campus, CEP 14884-900, Jaboticabal, SP, Brasil.

E-mail: bruno.barbosa@ posgrad.fcav.unesp.br.*Autor para correspondência.

${ }^{3}$ Departamento de Engenharia Rural, Universidade Estadual Paulista (UNESP) Campus de Jaboticabal, SP, Brasil.

${ }^{4}$ Departamento de Fitotecnia, Universidade Estadual Paulista (UNESP) Campus de Jaboticabal, SP, Brasil.
} 
Palavras-Chave: corda-de-viola, tecnologia de aplicação, bico de pulverização rotativo, volume de aplicação.

Abstract - The incorporation of tools related to the technology of application of pesticides is inevitable for a sustainable agriculture. We tested the efficiency in the control of Ipomoea nil in initial after-emergency with diuron + hexazinone, in two volumes of application $\left(20 \mathrm{ha}^{-1}\right.$ and 40 $\mathrm{L} \mathrm{ha}^{-1}$ ), with or without the addition of adjuvant, using peaks of rotating and hydraulically spraying, comparatively. Other two assays had been carried to compare the volumetric distribution of solution and the diameter of the drops produced for the hydraulical and rotating peaks. The percentage of necrosis in the leaves of $I$. nil plants increased progressively, in two volumes, with or without the use of adjuvants. The rotating peak did not differ from the hydraulically peak how much to the effectiveness. The use of rotary peak decreased coefficient of uniformity (SPAN) in relation to hydraulic peak, indicating the production of drops with greater uniformity. The distribution of the droplets produced by the rotary nozzle provided uneven distribution pattern in relation to the control peak (TT110.01 a $40 \mathrm{~L} \mathrm{ha}^{-1}$ ), due to lower volume of solution used. The peaks spacing was greater with the use of vegetable oil to the volume of $20 \mathrm{~L}$ $\mathrm{ha}^{-1}$. The association of the adjuvants to diuron + hexazinone provided to increase the diameter of the drops, with similar efficiency between the two tested volumes. The lower volume of application of diuron + hexazinone $\left(20 \mathrm{~L} \mathrm{ha}^{-1}\right)$ in $I$. nil control can be used, because it provided similar efficacy to the higher volume. The use of adjuvant organ silicone $(0.025 \% \mathrm{v} / \mathrm{v})$ and vegetable oil $(1.0 \% \mathrm{v} / \mathrm{v})$ may be recommended in the spray system with rotating tip allows for increasing the diameter of the droplets, reducing problems with drift, allowing the use of lower application volume (20 L ha-1), increasing the potential for overlapping droplets on the target.

Key-words: small wood rose, spraying technology, atomizer spraying nozzle, spraying volume.

\section{Introdução}

Com a racionalização da exploração agrícola a caminho da sustentabilidade e a incorporação de novas tecnologias na agricultura, tornam-se necessários o domínio, o conhecimento e o manejo dos principais fatores relacionados à produção, Neste sentido, a tecnologia de aplicação de produtos fitossanitários torna-se fundamental. Projetos e operações envolvendo a tecnologia de aplicação melhoraram de forma significativa nos últimos 50 anos. De acordo com Matuo (1990), a tecnologia de aplicação deve proporcionar uma aplicação de um produto biologicamente ativo sobre determinado alvo biológico, de maneira economicamente viável e com mínimo de contaminação ambiental.
Com o passar dos anos desenvolveramse pontas de pulverizações cada vez mais eficientes. O desenvolvimento de tecnologia como é o caso dos bicos rotativos capazes de aplicar gotas consideravelmente uniformes, a utilização de ventilação para a condução das gotas ao alvo, a adição de substâncias na calda capazes de alterar as características físicas, possibilitando menor deriva. Outra tecnologia é a utilização de carga eletrostática que proporciona um campo magnético e condiciona as gotas a serem atraídas pelas plantas que, por estarem aterradas, tendem a equilibrar as cargas do volume de calda com suas cargas, proporcionando eficiente deposição de gotas, inclusive na face abaxial das folhas (Fox et al., 2008). 
No Brasil, se tratando de tecnologia de aplicação, pesquisas são realizadas visando à caracterização do diâmetro de gota, distribuição, retenção e deposição do volume de aplicação e também pela caracterização de pontas de pulverização para aplicação de herbicidas e outros produtos fitossanitários. Entre os exemplos de empresas que atuam em tecnologia de aplicação encontram-se órgãos governamentais como a Embrapa Meio Ambiente (Pessoa \& Chaim, 1999; Pessoa et al., 1999; Chaim et al., 2002) e nas algumas instituições de ensino estadual ou federal (Ferreira et al., 2009; Silva et al., 2009; Lost et al., 2008; Silva et al., 2008; Bauer \& Raetano, 2000; Ruas et al., 2009; Magno Júnior, 2008).

O desenvolvimento de programas computacionais para estimar $\mathrm{o}$ fator de espalhamento, o diâmetro mediano volumétrico (dmv), o diâmetro mediano numérico (dmn), a uniformidade de gotas e o volume de deposição de calda foram realizados no final do século passado pelos pesquisadores Pessoa \& Chaim (1999) e Pessoa et al. (1999). Posteriormente, Chaim et al. (2002) propôs a avaliação computadorizada para avaliar o diâmetro das gotas e a densidade de gotas depositadas em papel sensível. Para isso, utilizaram imagens de gotas capturadas em papel hidrossensível e digitalizada para posterior análise em software, identificando as manchas do papel, contandoas e distribuindo-as em classes de diâmetro.

O controle químico é o principal método de controle utilizado para se manejar plantas daninhas. Entretanto, é preciso atingir o alvo com cobertura adequada pelo produto para que ocorra sucesso na aplicação fitossanitária, e para que isso ocorra é necessário conhecer as características de deposição proporcionadas pelo equipamento, produto e forma de aplicação (Ferreira et al, 2009). Bauer \& Raetano (2000) comentaram que o desenvolvimento da tecnologia de aplicação é indispensável para melhorar a eficiência de controle de insetos-praga, doenças e plantas daninhas.

Os produtos fitossanitários aplicados ao solo podem sofrer perdas acima de $33 \%$ por ocasião da endoderiva, pela má calibração e utilização de gotas de tamanhos inadequados. Além da endoderiva, a ocorrência significativa de ventos pode estar deslocando um grande volume de gotas pequenas para fora do alvo de aplicação, fenômeno conhecido como exoderiva (Scramin et al., 2002). Assim, objetivou-se estudar a eficácia e a qualidade de aplicação da mistura de diuron + hexazinone sobre plantas de Ipomoea nil, utilizando bico rotativo em dois volumes de aplicação, com e sem adjuvante.

\section{Material e métodos}

O experimento foi conduzido no Departamento de Fitossanidade da Faculdade de Ciências Agrárias e Veterinárias da Universidade Estadual de São Paulo, Campus de Jaboticabal, SP. Foram semeadas sementes de Ipomoea nil, em vasos com capacidade de 3 $\mathrm{L}$, preenchidos com substrato composto por solo, areia e esterco, na proporção 1:1:1, respectivamente. Após emergência, realizou-se o desbaste deixando cinco plantas por vaso.

Foi realizada uma aplicação em pósemergência inicial (2 a 3 folhas verdadeiras) de diuron + hexazinone $\left(1170+330 \mathrm{~g}\right.$ i.a. ha $\left.{ }^{-1}\right)$, em dois volumes de aplicação $\left(20 \mathrm{~L} \mathrm{ha}^{-1}\right.$ e $40 \mathrm{~L}$ $\mathrm{ha}^{-1}$ ), com ou sem a adição de um dos adjuvantes a calda de pulverização (espalhante siliconado a $0,025 \%$ e óleo vegetal a $1 \%$ ), utilizando-se um bico de pulverização rotativo (energia centrífuga) e um hidráulico (convencional).

$\mathrm{Na}$ ocasião da aplicação, a temperatura estava ao redor de $27^{\circ} \mathrm{C}$, a umidade relativa em $62 \%$ e a velocidade dos ventos de aproximadamente $3,5 \mathrm{~km} \mathrm{~h}^{-1}$. 
O delineamento utilizado foi o inteiramente casualizado com 4 repetições, em esquema fatorial $2 \times 3+2$. O primeiro fator representou o volume de aplicação $\left(20 \mathrm{~L} \mathrm{ha}^{-1} \mathrm{e}\right.$ $40 \mathrm{~L} \mathrm{ha}^{-1}$ ) e $\mathrm{o}$ segundo os adjuvantes espalhante siliconado $\left(\right.$ Silwett $^{\circledR}$ ) a $0,025 \% \mathrm{v} / \mathrm{v}$, óleo vegetal (VEGET'OIL) a $1 \%$ v/v e ausência de adjuvante. Em adição aos fatores, foram utilizadas duas testemunhas, uma representada pelo bico hidráulico TT11001 (convencional a $40 \quad$ L.ha $^{-1}$ ) e outra sem aplicação.

Foram avaliadas as porcentagens de controle (0-100\%) aos 7, 14 e 21 dias após a aplicação (DAA), através de observação visual com base na escala da SBCPD (1995), assim como a massa seca da parte aérea e das raízes aos 21 DAA. Para determinação massa seca, as plantas foram separadas em raízes e parte aérea, sendo posteriormente acondicionadas em sacos de papel e levadas à estufa de circulação forçada de ar à $65 \pm 5^{\circ} \mathrm{C}$, por um período de 72 horas. Posteriormente, as amostras secas foram pesadas em balança digital analítica de precisão, para obtenção das respectivas massas secas. Os dados de percentagem de controle e massa seca da parte aérea e raízes foram submetidos à análise de variância e as médias comparadas pelo teste Tukey $(\mathrm{P}<0,05)$.

No segundo experimento realizou-se a análise do espectro e caracterização da deposição de gotas, das mesmas caldas utilizadas para o controle de I. nil. A análise do espectro de gotas foi realizada de forma direta, utilizando-se um analisador de partículas Mastersizer $\mathrm{S}^{\circledR}$, Malvern Instruments Co., com lente focal de $300 \mathrm{~mm}$, comprimento de onda de laser de 0,5 a $900 \mathrm{~mm}$, que se baseia na difração da luz após o choque com as gotas, em que o diâmetro das gotas é proporcional ao ângulo do desvio sofrido pelo raio laser (Etheridge et al., 1999). No analisador, foram realizadas três leituras para cada tratamento, com intervalo entre as leituras de dois milisegundos, sendo realizadas 500 leituras por segundo. $\mathrm{O}$ acionamento do fluxo de calda foi realizado manualmente e o bico de pulverização foi posicionado próximo ao feixe do laser para que as gotas produzidas atravessassem o feixe, gerando os dados.

Avaliou-se o diâmetro das gotas, tal que $10 \%$ do volume aplicado sejam inferiores a esse valor (Dv 0,1); o diâmetro de gotas, tal que $50 \%$ do volume aplicado sejam inferiores ou superiores a esse valor (Dv 0,5); o diâmetro de gotas, tal que $90 \%$ do volume aplicado sejam inferiores a esse valor (Dv 0,9); a coeficiente de uniformidade (AR) e a porcentagem do volume aplicado cujas gotas possuam diâmetro inferior a $100 \mu \mathrm{m}(\mathrm{Dv}<100$ $\mu \mathrm{m})$.

O coeficiente de uniformidade é dado por:

$$
\mathrm{COEF}=\frac{\mathrm{Dv} 0,9-\mathrm{Dv} 0,1}{\mathrm{Dv} 0,5}
$$

O delineamento utilizado foi $\mathrm{o}$ inteiramente casualizado com 02 repetições, em esquema fatorial $2 \times 3+1$. O primeiro fator representou o volume de aplicação $\left(20 \mathrm{~L} \mathrm{ha}^{-1} \mathrm{e}\right.$ $40 \mathrm{~L} \mathrm{ha}^{-1}$ ), o segundo os adjuvantes (espalhante siliconado a $0,025 \% \mathrm{v} / \mathrm{v}$, óleo vegetal a $1 \% \mathrm{v} / \mathrm{v}$ e ausência de adjuvante) e uma testemunha representada pelo bico hidráulico convencional (TT11001 a 40 L.ha $^{-1}$ ). Os dados de Dv 0,1, Dv 0,5 , Dv 0,9, porcentagem de gotas com diâmetro igual ou inferior a $100 \mu \mathrm{m}$ e a coeficiente de uniformidade foram submetidos à análise de variância e as médias comparadas pelo teste Tukey $(\mathrm{P}<0,05)$. Os dados originais de percentagem foram transformados em $\operatorname{Arcoseno}(\sqrt{ }(\mathrm{P}<100 \mu / 100))$ para a análise estatística. Foram utilizadas apenas 2 repetições devido ao coeficiente de variação extremamente baixo proporcionado pelo equipamento analisador de partículas. 
Para a determinação do perfil de distribuição volumétrica das caldas pulverizadas (terceiro experimento), utilizou-se o bico hidráulico com ponta TT11001 como testemunha. Utilizou-se mesa de provas construída de acordo com as especificações da ORGANIZACIÓN MUNDIAL DE LA SALUD (1976) para testes de bicos, constituída de 67 canaletas de metal corrugado com 2,5 cm de largura cada, totalizando 167,5 $\mathrm{cm}$ de largura por $100 \mathrm{~cm}$ de comprimento. As canaletas possuem inclinação de $15^{\circ}$ para o líquido pulverizado escoar até tubos de ensaio graduados, utilizados como coletores. O bico hidráulico foi posicionado verticalmente à altura da mesa de $50 \mathrm{~cm}$, acima da canaleta $\mathrm{n}^{\circ}$ 33 , no centro da mesa de provas.

Para a avaliação do bico rotativo utilizou-se o método de papéis hidrossensíveis, devido ao maior ângulo do jato de pulverização produzido que não pode ser realizado em mesa de provas. Os papéis foram dispostos um ao lado do outro, espaçados a $20 \mathrm{~cm}$, totalizando uma faixa de $2,40 \mathrm{~m}$, sendo realizadas três repetições. Após a passagem do aplicador, os papéis foram retirados e colocados em sacos de papel identificados, com o auxílio de luvas plásticas, com a finalidade de mantê-los íntegros e fora da exposição de umidade. Após a aplicação, os papéis hidrossensíveis foram digitalizados em escâner de mesa, com resolução de 300 dpi, as imagens obtidas foram analisadas no software QUANT v 0.1, para quantificação da porcentagem da área coberta pelas gotas (Vale et al., 2001).

Para a determinação do espaçamento de trabalho entre bicos, foram utilizados os coeficientes de variação, que dentro do limite aceitável na literatura, assumiu-se $10 \%$, de acordo com Wolf \& Smith (1979) e FAO (1997).

Os dados obtidos foram submetidos à análise de variância e as médias comparadas pelo teste Tukey $(\mathrm{P}<0,05)$. Realizou-se ainda a análise de regressão a fim de se verificar qual o modelo de melhor ajuste aos dados.

\section{Resultados e discussão}

$O$ percentual de necrose foliar aumentou progressivamente, nos dois volumes, independentemente da utilização de adjuvante (Tabela 1). O bico rotativo proporcionou controle semelhante ao obtido com a ponta hidráulica, em todas as avaliações, para todos os tratamentos que receberam aplicação do herbicida. Esse resultado sugere que a ponta para uso na aplicação de diuron+hexazinone, pode ser rotativa ou hidráulica.

Quanto ao volume de calda, é possível afirmar que a eficácia da ponta rotativa utilizando $20 \mathrm{~L} \mathrm{ha}^{-1}$ foi tão eficiente quanto 40 $\mathrm{L} \mathrm{ha}^{-1}$, possibilitando a aplicação da menor volume de calda, e consequentemente, diminuindo impactos econômicos e ambientais (Tabela 1). Quando se aplica volume acima do necessário, além dos maiores custos gerados para aquisição da quantidade necessária de ingrediente ativo, existe o custo operacional que é consideravelmente elevado pelo menor rendimento na aplicação, com consequentes reabastecimentos do tanque do pulverizador. Problemas com deposição de gotas fora do alvo também se intensificam, potencializando a deriva, bem como a deposição de gotas do herbicida em plantas que não deveriam ser dessecadas. 
Tabela 1 - Análise de variância em esquema fatorial 2 x $3+2$ da porcentagem de controle de Ipomoea nil, pulverizadas com herbicida diuron + hexazinone em dois volumes, associado ou não a utilização de adjuvantes. Jaboticabal, SP. 2009.

\begin{tabular}{|c|c|c|c|}
\hline \multirow[t]{2}{*}{ Fatores } & \multicolumn{3}{|c|}{ Períodos de Avaliação Dias Após a Aplicação } \\
\hline & 7 DAA & $14 \mathrm{DAA}$ & $21 \mathrm{DAA}$ \\
\hline & \multicolumn{3}{|c|}{ Volume de calda } \\
\hline $20 \mathrm{~L}^{2} \mathrm{ha}^{-1}$ & $23,75 \mathrm{a}$ & $79,17 \mathrm{a}$ & $99,58 \mathrm{a}$ \\
\hline 40 L.ha $^{-1}$ & $20,42 \mathrm{a}$ & $67,08 \mathrm{a}$ & $97,08 \mathrm{a}$ \\
\hline Teste F & $0,44^{\mathrm{NS}}$ & $3,73^{\mathrm{NS}}$ & $1,79^{\mathrm{NS}}$ \\
\hline \multirow[t]{2}{*}{ DMS $(5 \%)$} & 7,97 & 9,74 & 4,68 \\
\hline & \multicolumn{3}{|c|}{ Adjuvantes } \\
\hline Ausente & $25,63 \mathrm{a}$ & $67,50 \mathrm{a}$ & $99,37 \mathrm{a}$ \\
\hline Silwett $^{\circledR 1}$ & $21,88 \mathrm{a}$ & $70,00 \mathrm{a}$ & $95,62 \mathrm{a}$ \\
\hline VEGET’OIL ${ }^{\circledR 2}$ & $18,75 \mathrm{a}$ & $81,87 \mathrm{a}$ & $100,00 \mathrm{a}$ \\
\hline Teste F & $0,44^{\mathrm{NS}}$ & $2,45^{\mathrm{NS}}$ & $2,65^{\mathrm{NS}}$ \\
\hline DMS $(5 \%)$ & 11,81 & 14,43 & 6,93 \\
\hline & \multicolumn{3}{|c|}{ Tratamentos adicionais } \\
\hline Convencional (ponta hidráulica) & $20,00 \mathrm{a}$ & $90,00 \mathrm{a}$ & $100,00 \mathrm{a}$ \\
\hline \multirow[t]{2}{*}{ Testemunha } & $00,00 \mathrm{~b}$ & $00,00 \mathrm{~b}$ & $0,00 \mathrm{~b}$ \\
\hline & \multicolumn{3}{|c|}{ Teste $\mathrm{F}$} \\
\hline Teste F (Volume x Adjuvantes) & $0,91^{\mathrm{NS}}$ & $1,48^{\mathrm{NS}}$ & $4,37^{*}$ \\
\hline Teste F (Fatorial) & $0,63^{\mathrm{NS}}$ & $2,32^{\mathrm{NS}}$ & $3,17^{*}$ \\
\hline Teste $\mathrm{F}$ (Testemunha $\mathrm{x}$ Fatorial) & $13,00^{* *}$ & $24,05^{* *}$ & $349,33^{* *}$ \\
\hline C.V. & 40,05 & 21,14 & 7,24 \\
\hline
\end{tabular}

*Diferença estatística ao nível de 5\% de probabilidade; **Diferença estatística ao nível de $1 \%$ de probabilidade; ${ }^{\mathrm{NS}}$ Diferença não significativa a 5\% de probabilidade; Dados originais comparados pelo teste de Tukey. Médias seguidas pelas mesmas letras não diferem significativamente entre si; ${ }^{1}$ Adjuvante Siliconado a $0,025 \%$; ${ }^{2}$ Adjuvante Óleo Vegetal a $1 \% ;{ }^{3}$ Dados originais transformados em $\operatorname{Arcoseno}(\sqrt{ }(\mathrm{P}<100 \mu / 100))$

A adição do adjuvante Silwett $^{\circledR}$ ao volumes de calda para Silwett ${ }^{\circledR}$, porém, ao herbicida prejudicou o controle da planta contrário do ocorrido para $20 \mathrm{~L} \mathrm{ha}^{-1}$, a daninha em relação ao óleo vegetal aplicação de $40 \mathrm{~L} \mathrm{ha}^{-1}$ não sofreu qualquer VEGET'OIL $^{\circledR}$ e a não adição de adjuvante, interferência da utilização de nenhum dos quando utilizado o menor volume de calda (20 adjuvantes testados (Tabela 2).

$\left.\mathrm{L} \mathrm{ha}{ }^{-1}\right)$. Houve interação significativa entre os

Tabela 2. Desdobramento da interação Volume vs. Adjuvante para o percentual de controle de Ipomoea nil, 21 dias após a aplicação do herbicida diuron + hexazinone em dois volumes, associado ou não a utilização de adjuvantes. Jaboticabal, SP. 2009.

\begin{tabular}{|c|c|c|c|c|}
\hline \multirow{2}{*}{ Concentração } & \multicolumn{3}{|c|}{ Adjuvantes } & \multirow{2}{*}{ Teste F } \\
\hline & Ausente & Silwett $^{\circledR 1}$ & VEGET'OIL $^{\circledR 2}$ & \\
\hline $20 \mathrm{~L} \mathrm{ha}^{-1}$ & $100,00 \mathrm{Aa}$ & $91,25 \mathrm{Bb}$ & $100,00 \mathrm{Aa}$ & $6,57^{* *}$ \\
\hline $40 \mathrm{~L} \mathrm{ha}^{-1}$ & $98,75 \mathrm{Aa}$ & $100,00 \mathrm{Aa}$ & $100,00 \mathrm{Aa}$ & $0,45^{\mathrm{NS}}$ \\
\hline Teste F & $0,68^{\mathrm{NS}}$ & $9,86^{* *}$ & $0,00^{\mathrm{NS}}$ & - \\
\hline
\end{tabular}

**Diferença estatística ao nível de $1 \%$ de probabilidade; ${ }^{\text {NS }}$ Diferença não significativa a $5 \%$ de probabilidade. Dados originais comparados pelo teste de Tukey. Médias seguidas pelas mesmas letras não diferem significativamente entre si. ${ }^{1}$ Adjuvante Silwett a $0,025 \% ;{ }^{2}$ Adjuvante Óleo Vegetal a $1 \% ;{ }^{3}$ Dados originais transformados em Arcoseno( $\sqrt{ }(\mathrm{P}$ $<100 \mu / 100)$ ) para o teste de comparação 
Os valores de massa seca da parte aérea e raízes apresentaram comportamento semelhante, sendo que, ambos proporcionaram resultados coerentes aos de controle. Ou seja, todos tratamentos herbicidas se comportaram de maneira semelhante, não diferindo significativamente entre si. As médias das duas variáveis de desenvolvimento vegetal, para as plantas que não receberam herbicidas (testemunha), foram significativamente maiores (Tabela 3), evidenciando a eficácia dos tratamentos.

Tabela 3 - Análise de variância em esquema fatorial 2 × $3+2$ do efeito da pulverização com herbicida diuron + hexazinone nos valores de massa seca da parte aérea e raízes de Ipomoea nil, em dois volumes, associado ou não a utilização de adjuvantes. Jaboticabal, SP. 2009.

\begin{tabular}{|c|c|c|}
\hline \multirow{2}{*}{ Fatores } & \multicolumn{2}{|c|}{ Massa seca $(\mathrm{g})-\mathrm{MS}$} \\
\hline & MS aérea & MS raízes \\
\hline & \multicolumn{2}{|c|}{ Concentração } \\
\hline $20 \mathrm{~L} \mathrm{ha}^{-1}$ & $0,07 \mathrm{a}$ & $0,15 \mathrm{a}$ \\
\hline $40 \mathrm{~L} \mathrm{ha}^{-1}$ & $0,06 \mathrm{a}$ & $0,14 \mathrm{a}$ \\
\hline Teste F & $0,04^{\mathrm{NS}}$ & $0,19^{\mathrm{NS}}$ \\
\hline \multirow[t]{2}{*}{ DMS $(5 \%)$} & 0,07 & 0,05 \\
\hline & \multicolumn{2}{|c|}{ Adjuvante } \\
\hline Ausente & $0,05 \mathrm{a}$ & $0,13 \mathrm{a}$ \\
\hline Silwett $^{\circledR 1}$ & $0,08 \mathrm{a}$ & $0,17 \mathrm{a}$ \\
\hline VEGET'OIL ${ }^{\circledR 2}$ & $0,07 \mathrm{a}$ & $0,15 \mathrm{a}$ \\
\hline Teste F & $0,16^{\mathrm{NS}}$ & $0,96^{\mathrm{NS}}$ \\
\hline \multirow[t]{2}{*}{ DMS $(5 \%)$} & 0,11 & 0,07 \\
\hline & \multicolumn{2}{|c|}{ Tratamentos adicionais } \\
\hline Convencional & $0,08 \quad \mathrm{~b}$ & $0,12 \mathrm{~b}$ \\
\hline \multirow[t]{2}{*}{ Testemunha } & $0,54 \mathrm{a}$ & $0,38 \mathrm{a}$ \\
\hline & Teste F & \\
\hline Volume x Adjuvantes & $0,49^{\mathrm{NS}}$ & $0,79^{\mathrm{NS}}$ \\
\hline Fatorial & $0,27^{\mathrm{NS}}$ & $0,73^{\mathrm{NS}}$ \\
\hline Testemunha $\mathrm{x}$ Fatorial & $45,53 * *$ & $18,14 * *$ \\
\hline C.V. & 69,57 & 32,55 \\
\hline
\end{tabular}

**Diferença estatística ao nível de $1 \%$ de probabilidade; ${ }^{\text {NS }}$ Diferença não significativa a $5 \%$ de probabilidade. Dados originais comparados pelo teste de Tukey. Médias seguidas pelas mesmas letras não diferem significativamente entre si. ${ }^{1}$ Adjuvante Silwett a $0,025 \% ;{ }^{2}$ Adjuvante Óleo Vegetal a $1 \%$

O bico rotativo não diferiu do bico hidráulico quanto ao efeito do herbicida nas plantas, podendo ser utilizado para favorecer a aplicação de menores volumes de aplicação (Tabelas 1 e 2).

$\mathrm{O}$ uso dos adjuvantes siliconado $\left(\right.$ Silwett $\left.^{\circledR}\right) \quad(0,025 \%)$ e Óleo Vegetal $(1, \%)$, proporcionou aumento para os Diâmetro Volumétrico DV0,1, DV 0,5 e DV 0,9 em relação a ausência de aplicação, com diferença mais acentuada em relação ao volume de calda de $20 \mathrm{~L} \mathrm{ha}^{-1}$ (Tabelas 4, 5, 6 e 7).

Quando bicos hidráulicos são usados, uma ampla variação de gotas emitidas resulta em consideráveis diferenças na relação cargamassa, assim a trajetória de gotas grandes, com menores valores para esta relação, não é significativamente afetada, e a deposição nem sempre é aumentada. Deste modo, os melhores resultados são alcançados com gotas pequenas 
e, conseqüentemente, menores volumes de aplicação (Mathews, 1989). A não utilização dos adjuvantes refletiu em diminuição do diâmetro médio das gotas para cada faixa de DV $(0,1,0,5$ e 0,9$)$, sendo para ponta hidráulica, o diâmetro das gotas foi significativamente menor, proporcionando elevado número de gotas menores que $100 \mu \mathrm{e}$ aumento da deriva (Tabela 4 e 8 ).

O Coeficiente de uniformidade aumentou significativamente quando aplicada $\mathrm{o}$ maior volume de calda (40 L.ha ${ }^{-1}$ ), sobretudo ao adicionar-se qualquer um dos adjuvantes testados (Tabela 4 e 8). Maiores volumes de calda proporcionam maior AR e, consequentemente, menor controle da uniformidade de gotas. Essa não é uma característica interessante na tecnologia de aplicação, uma vez que diminui a capacidade de controle sobre as gotas que vão efetivamente atingir $o$ alvo.

Tabela 4 - Análise de variância em esquema fatorial 2 × $3+2$ dos valores de diâmetro médio das gotas produzidas pelo bico rotativo, DV $(0,1,0,5$ e 0,9$)$, do percentual de gotas menores que 100 $\mu \mathrm{m}$ e da Coeficiente de uniformidade (AR), comparativamente ao bico hidráulico (TT11001). Jaboticabal, 2009.

\begin{tabular}{|c|c|c|c|c|c|}
\hline \multirow{2}{*}{ Fatores } & \multicolumn{5}{|c|}{ Variáveis } \\
\hline & DV 0,1 & DV 0,5 & DV0,9 & $\mathrm{P}<100 \mu \mathrm{m}(\%)^{3}$ & COEF \\
\hline & \multicolumn{5}{|c|}{ Concentração } \\
\hline $20 \mathrm{Lha}^{-1}$ & $150,61 \mathrm{a}$ & $237,12 \mathrm{~b}$ & $369,92 \mathrm{~b}$ & $1,05 \mathrm{a}$ & $0,89 \mathrm{~b}$ \\
\hline $40 \mathrm{~L} \mathrm{ha}^{-1}$ & $153,39 \mathrm{a}$ & $259,91 \mathrm{a}$ & $414,89 \mathrm{a}$ & $1,11 \mathrm{a}$ & $0,99 \mathrm{a}$ \\
\hline Teste F (Volume) & $0,91^{\mathrm{NS}}$ & $22,73^{* *}$ & $29,22^{* *}$ & $0,28^{\mathrm{NS}}$ & $21,18^{* * * *}$ \\
\hline \multirow[t]{2}{*}{$\operatorname{DMS}(5 \%)$} & 6,91 & 11,30 & 19,67 & 0,02 & 0,06 \\
\hline & \multicolumn{5}{|c|}{ Adjuvantes } \\
\hline Ausente & $141,79 \mathrm{~b}$ & $214,55 \mathrm{c}$ & $326,68 \mathrm{c}$ & $1,23 \mathrm{a}$ & $0,85 \mathrm{~b}$ \\
\hline Silwett ${ }^{\circledR 1}$ & 153,63 a & $255,64 \mathrm{~b}$ & $408,01 \mathrm{~b}$ & $1,09 \mathrm{a}$ & $0,96 \mathrm{a}$ \\
\hline VEGET'OIL $^{\circledR 2}$ & $160,57 \mathrm{a}$ & $275,35 \mathrm{a}$ & $442,52 \mathrm{a}$ & $0,92 \mathrm{a}$ & $1,01 \mathrm{a}$ \\
\hline Teste F (Adjuvantes) & $14,09^{* *}$ & $56,14^{* *}$ & $68,15^{* *}$ & $1,20^{\mathrm{NS}}$ & $15,80^{* *}$ \\
\hline DMS $(5 \%)$ & 10,53 & 17,24 & 30,01 & 0,03 & 0,08 \\
\hline \multirow[t]{2}{*}{ Convencional (ponta hidráulica) } & 73,06 & 168,30 & 17,40 & 21,54 & 1,45 \\
\hline & \multicolumn{5}{|c|}{ Teste $\mathrm{F}$} \\
\hline Teste F (Volume x Adjuvantes) & $21,33^{* *}$ & $104,45^{* *}$ & $180,02^{* *}$ & $2,87^{\mathrm{NS}}$ & $68,91^{* *}$ \\
\hline Teste F (Fatorial) & $14,35^{* *}$ & $68,78^{* *}$ & $105,11^{* *}$ & $1,68^{\mathrm{NS}}$ & $38,12^{* * *}$ \\
\hline Teste F (Conv.x Fatorial) & $417,48^{* *}$ & $160,83^{* *}$ & $60,79^{* *}$ & $1172,14^{* *}$ & $275,12^{* *}$ \\
\hline C.V. & 3,6 & 3,5 & 3,8 & 1,0 & 4,0 \\
\hline
\end{tabular}

**Diferença estatística ao nível de $1 \%$ de probabilidade; ${ }^{\text {NS }}$ Diferença não significativa a $5 \%$ de probabilidade. Dados originais comparados pelo teste de Tukey. Médias seguidas pelas mesmas letras não diferem significativamente entre si.; ${ }^{1}$ Adjuvante Silwett a $0,025 \% ;{ }^{2}$ Adjuvante Óleo Vegetal a $1 \% ;{ }^{3}$ Dados originais transformados em Arcoseno $(\sqrt{ }(\mathrm{P}$ $<100 \mu \mathrm{m} / 100))$ 
Tabela 5 - Desdobramento da interação Volume vs. Adjuvante para DV 0,1 das gotas produzidas pelo bico rotativo. Jaboticabal, SP. 2009.

\begin{tabular}{ccclc}
\hline \multirow{2}{*}{ Concentração } & \multicolumn{3}{c}{ Adjuvantes } & \multirow{2}{*}{ Teste F } \\
\cline { 2 - 4 } & Ausente & Silwett $^{\circledR 1}$ & VEGET'OIL $^{\circledR 2}$ & \\
\hline $20 \mathrm{~L} \mathrm{ha}^{-1}$ & $136,9 \mathrm{Ba}$ & $142,6 \mathrm{Ba}$ & $172,2 \mathrm{Ab}$ & $28,0^{* * *}$ \\
$40 \mathrm{~L} \mathrm{ha}^{-1}$ & $146,6 \mathrm{Ba}$ & $164,6 \mathrm{Ab}$ & $148.9 \mathrm{Ba}$ & $7,4^{*}$ \\
\hline Teste F & $3,7^{\text {NS }}$ & $18,7^{* * *}$ & $21,2^{* * *}$ & -
\end{tabular}

*Diferença estatística ao nível de 5\% de probabilidade; **Diferença estatística ao nível de 1\% de probabilidade

${ }^{N S}$ Diferença não significativa a $5 \%$ de probabilidade. Dados originais comparados pelo teste de Tukey. Médias seguidas pelas mesmas letras não diferem significativamente entre si. ${ }^{1}$ Adjuvante a $0,025 \% ;{ }^{2}$ Adjuvante a $1 \%$

Tabela 6 - Desdobramento da interação Volume vs. Adjuvante para DV 0,5 das gotas produzidas pelo bico rotativo. Jaboticabal, SP. 2009.

\begin{tabular}{ccccc}
\hline \multirow{2}{*}{ Concentração } & \multicolumn{3}{c}{ Adjuvantes } & \multirow{2}{*}{ Teste F } \\
\cline { 2 - 4 } & Ausente & Silwett $^{\circledR 1}$ & VEGET'OIL $^{\circledR 2}$ & \\
\hline $20 \mathrm{~L} \mathrm{ha}^{-1}$ & $188,96 \mathrm{Bb}$ & $311,55 \mathrm{Ba}$ & $210,85 \mathrm{Ab}$ & $124,66^{* * *}$ \\
$40 \mathrm{~L} \mathrm{ha}^{-1}$ & $240,14 \mathrm{Ba}$ & $239,16 \mathrm{Ab}$ & $300,44 \mathrm{Ba}$ & $35,93^{* *}$ \\
\hline Teste F & $38,19^{* * *}$ & $76,41^{* * *}$ & $117,03^{* *}$ & - \\
\hline
\end{tabular}

**Diferença estatística ao nível de $1 \%$ de probabilidade Dados originais comparados pelo teste de Tukey. Médias seguidas pelas mesmas letras não diferem significativamente entre si. ${ }^{1}$ Adjuvante a $0,025 \% ;{ }^{2}$ Adjuvante a $1 \%$.

Tabela 7 - Desdobramento da interação Volume vs. Adjuvante para DV 0,9 das gotas produzidas pelo bico rotativo. Jaboticabal, SP. 2009.

\begin{tabular}{ccclc}
\hline \multirow{2}{*}{ Concentração } & \multicolumn{3}{c}{ Adjuvantes } & \multirow{2}{*}{ Teste F } \\
\cline { 2 - 4 } & Ausente & Silwett & \\
\hline $20 \mathrm{~L} \mathrm{ha}^{-1}$ & $279,75 \mathrm{Bb}$ & $303,44 \mathrm{Ba}$ & $526,58 \mathrm{Ab}$ & $178,67^{* * *}$ \\
$40 \mathrm{~L} \mathrm{ha}^{-1}$ & $373,62 \mathrm{Ba}$ & $512,58 \mathrm{Ab}$ & $358,47 \mathrm{Ba}$ & $69,50^{* *}$ \\
\hline Teste F & $42,45^{* *}$ & $136,13^{* * *}$ & $210,67^{* * *}$ & -
\end{tabular}

**Diferença estatística ao nível de 1\% de probabilidade. Dados originais comparados pelo teste de Tukey. Médias seguidas pelas mesmas letras não diferem significamente entre si. ${ }^{1}$ Adjuvante a $0,025 \% ;{ }^{2}$ Adjuvante a $1 \%$.

Tabela 8 - Desdobramento da interação Volume vs. Adjuvante para a variável coeficiente de uniformidade (AR) das gotas produzidas pelo bico rotativo. Jaboticabal, SP. 2009.

\begin{tabular}{cllll}
\hline \multirow{2}{*}{ Concentração } & \multicolumn{3}{c}{ Adjuvantes } & \multirow{2}{*}{ Teste F } \\
\cline { 2 - 4 } & Ausente & Silwett & \\
\hline $20 \mathrm{~L} \mathrm{ha}^{-1}$ & $0,76 \mathrm{Bb}$ & $1,14 \mathrm{Ba}$ & $0,76 \mathrm{Ab}$ & $58,21^{* *}$ \\
$40 \mathrm{~L} \mathrm{ha}^{-1}$ & $0,94 \mathrm{Ba}$ & $0,88 \mathrm{Ab}$ & $1,16 \mathrm{Ba}$ & $26,51^{* *}$ \\
\hline Teste $\mathrm{F}$ & $21,53^{* *}$ & $41,69^{* *}$ & $95,79^{* *}$ & - \\
\hline
\end{tabular}

**Diferença estatística ao nível de $1 \%$ de probabilidade. Dados originais comparados pelo teste de Tukey. Médias seguidas pelas mesmas letras não diferem significativamente entre si. ${ }^{1}$ Adjuvante a $0,025 \% ;{ }^{2}$ Adjuvante a $1 \%$.

Ao observarmos os valores de (TT11001) proporcionou elevado potencial de percentual de gotas menores que $100 \mu \mathrm{m}$ deriva, pois o percentual de gotas menores que podemos inferir que a ponta hidráulica $100 \mu$ é consideravelmente superior a do bico 
rotativo. Essas gotas, devido à sua pequena massa, possuem pouca energia cinética, o que faz com que sua coleta pelo alvo seja reduzida e também a deriva seja bastante acentuada (Chaim, 1984). Os menores valores de coeficiente de uniformidade (COEF) produzidas pelo bico rotativo revelam a superior uniformidade das gotas proporcionadas pelo mesmo (Tabela 4 e 8 ).

Houve aumento no diâmetro médio das gotas (DV 0,5), tanto com a aplicação no maior volume (40 L.ha ${ }^{-1}$ ), quanto quando adicionouse um dos adjuvantes, principalmente quando este se tratava do óleo vegetal (Tabela 4 e 5). O percentual de gotas menores que $100 \mu$ não foi influenciado por nenhum dos tratamentos, exceto quando compara-se os resultados obtidos com os bicos rotativo e hidráulico (Tabela 4). A coeficiente de uniformidade aumentou com o aumento do volume (40 L.ha
${ }^{1}$ ), diminuindo assim a uniformidade do diâmetro volumétrico das gotas (Tabela 8).

A distribuição das gotas produzidas pelo bico rotativo, de maneira geral, resultou em padrão de distribuição desuniforme, devido aos baixos volumes de aplicação, haja vista que a distribuição das gotas foi mais uniforme quando foi aplicado 40 L.ha $^{-1}$. Verifica-se que o mesmo não ocorreu para a testemunha (TT11001), que utilizou volume alto de aplicação, resultando em padrão de distribuição mais uniforme (Figura 1). Volumes alto de aplicação proporcionaram maiores quantidades de gotas de pequeno diâmetro (menores que $100 \mu \mathrm{m})$.

Na Figura 2, observam-se as equações de regressão para a determinação do espaçamento entre bicos, em função do coeficiente de variação.
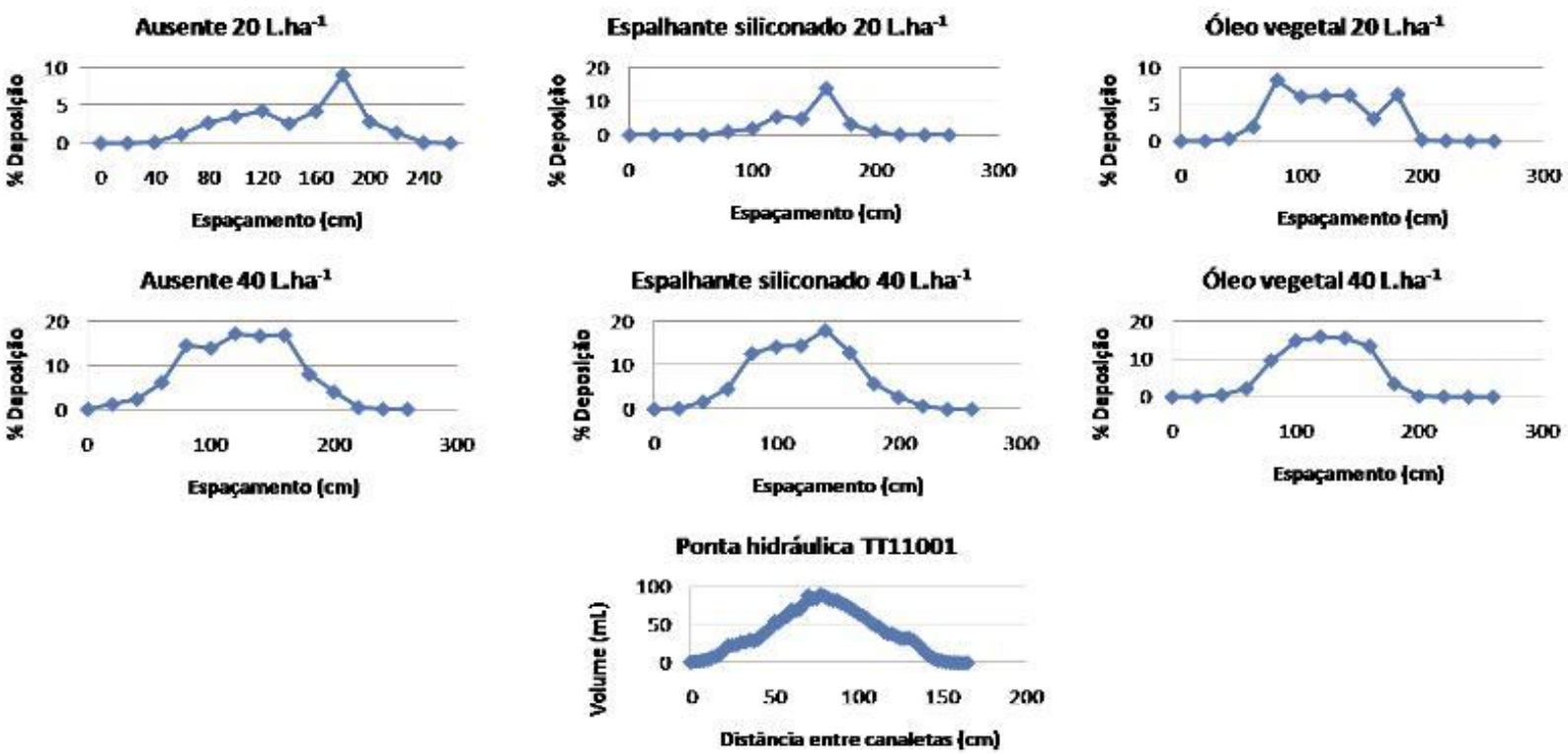

Figura 1 - Perfil de distribuição volumétrica sem adjuvante com volume de aplicação de $20 \mathrm{~L} \mathrm{ha}^{-}$ 1 e $40 \mathrm{~L} \mathrm{ha}^{-1}$, com o adjuvante siliconado e volume de $20 \mathrm{~L} \mathrm{ha}^{-1}$ e $40 \mathrm{~L} \mathrm{ha}^{-1}$, com óleo vegetal e volume de $20 \mathrm{~L} \mathrm{ha}^{-1}$ e $40 \mathrm{~L} \mathrm{ha}^{-1}$ e testemunha convencional utilizando-se bico hidráulico (TT11001). Jaboticabal, 2009. 


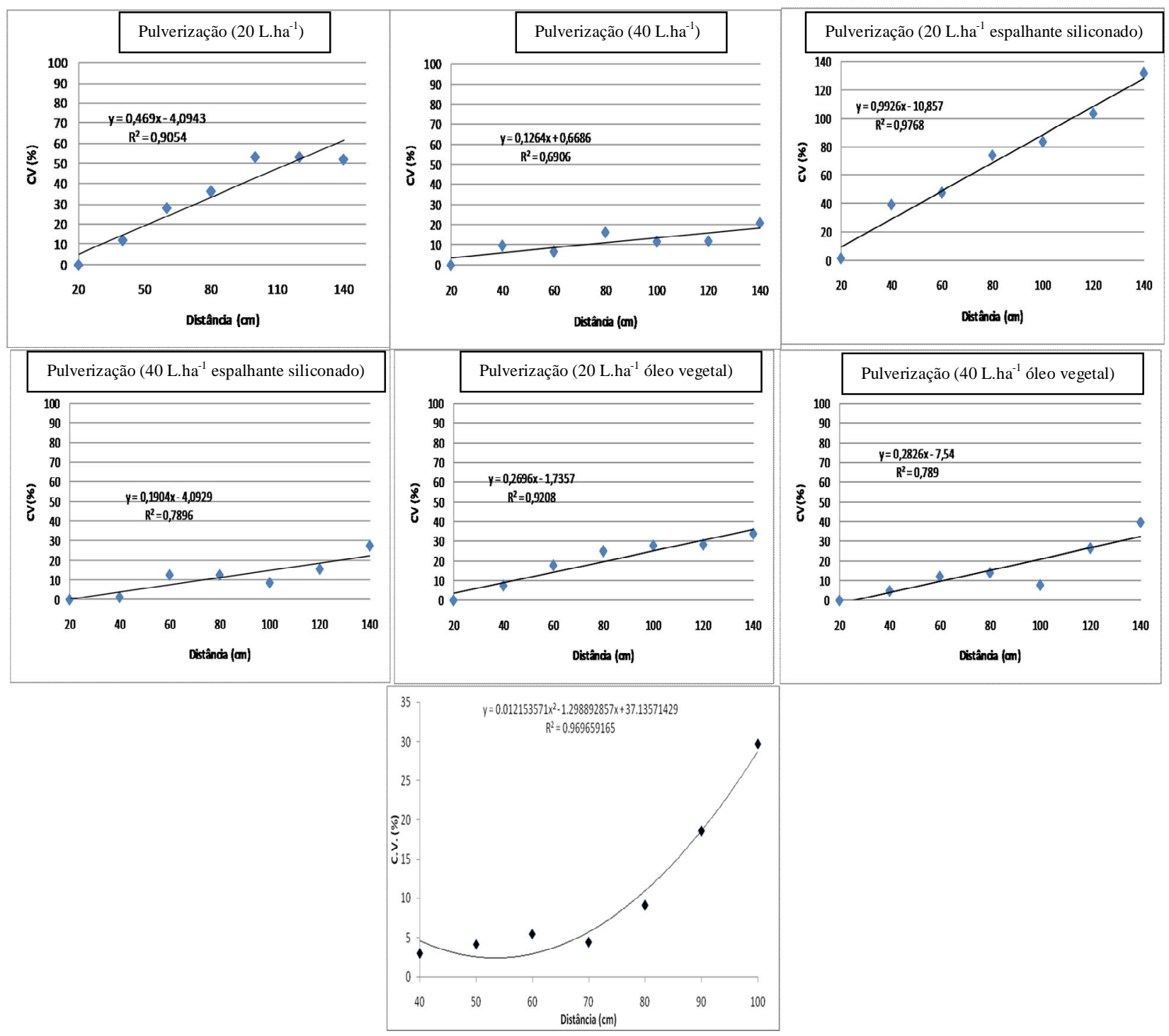

Figura 2 - Equações de regressão para a determinação do espaçamento entre bicos, em função do coeficiente de variação para volume de aplicação de $20 \mathrm{~L} \mathrm{ha}^{-1}$ e $40 \mathrm{~L} \mathrm{ha}^{-1}$, com adição de adjuvante siliconado e volume de $20 \mathrm{~L} \mathrm{ha}^{-1}$ e $40 \mathrm{~L} \mathrm{ha}^{-1}$, com óleo vegetal e volume de $20 \mathrm{~L} \mathrm{ha}^{-1} \mathrm{e}$ $40 \mathrm{~L} \mathrm{ha}^{-1}$ e testemunha convencional utilizando bico hidráulico (TT11001). Jaboticabal, 2009.

Como o experimento foi desenvolvido em condições de laboratório, os espaçamentos entre pontas apresentadas na Tabela 9 foram calculados em função dos coeficientes de variação aceitáveis na literatura, portanto, inferiores a $10 \%$. Em condições de campo, tais valores tendem a aumentar, seja pelas condições climáticas inerentes por ocasião da aplicação ou mesmo pelos movimentos desordenados da barra de pulverização (Perecin et al. 1998). 
Tabela 9 - Espaçamento aceitável entre bicos (com C.V. de 10\%) utilizando-se diferentes volumes de aplicação e adjuvantes. Jaboticabal, 2009.

\begin{tabular}{|c|c|c|}
\hline \multirow{3}{*}{ Tratamentos } & \multicolumn{2}{|c|}{ Volume } \\
\hline & $\mathrm{ha}^{-1} 20 \mathrm{~L}$ & $40 \mathrm{~L} \mathrm{ha}^{-1}$ \\
\hline & \multicolumn{2}{|c|}{ Espaçamento $(\mathrm{cm})$} \\
\hline Ausência de adjuvante & 29,14 & 91,30 \\
\hline Espalhante Siliconado Silwett ${ }^{\circledR}$ & 22.00 & 85,80 \\
\hline Óleo Vegetal VEGET’OIL ${ }^{\circledR}$ & 40,60 & 79,50 \\
\hline $\begin{array}{l}\text { Testemunha Convencional } \\
\left(\text { TT11001 40 L.ha }{ }^{-1}\right)\end{array}$ & \multicolumn{2}{|c|}{78,40} \\
\hline
\end{tabular}

O espaçamento entre bicos foi maior com o uso de óleo vegetal para o volume de aplicação de $20 \mathrm{~L} \mathrm{ha}^{-1}$. No volume de aplicação de $40 \mathrm{~L}$ ha $^{-1}$ os espaçamentos foram semelhantes (Tabela 9). O uso dos adjuvantes proporcionou maior formação de gotas DV0,1, 0,5 e 0,9 , sendo que, quando utilizado o óleo vegetal VEGET'OIL ${ }^{\circledR}$, o DV foi maior na concentração $20 \mathrm{~L} \mathrm{ha}^{-1}$. No caso do espalhante siliconado Silwett ${ }^{\circledR}$, os maiores DV foram formados na concentração de $40 \mathrm{~L} \mathrm{ha}^{-1}$. Essas gotas de maior massa alcançam maiores distâncias, possibilitando maior espaçamento entre pontas, com cobertura satisfatória (Tabelas 4, 5, 6 e 7).

\section{Conclusões}

O volume de aplicação de $20 \mathrm{~L} \mathrm{ha}^{-1}$ pode ser utilizado com o herbicida diuron+hexazinone no controle de Ipomoea nil, pois proporcionou mesma eficácia ao volume maior, possibilitando menores impactos ambientais e sócios econômicos;
Os adjuvantes organo siliconado $(0,025 \%)$ e óleo vegetal $(1,0 \%)$ não afetaram a porcentagem de controle de Ipomoea nil., mas podem ser recomendados em sistema de pulverização com bico rotativo por permite aumento do diâmetro médio das gotas, com redução do potencial de deriva, principalmente para o volume de aplicação de $20 \mathrm{~L} \mathrm{ha}^{-1}$.

\section{Agradecimentos}

Ao Técnico Agrícola Gilson José Leite, funcionário do Departamento de Fitossanidade, e ao Engenheiro Agrônomo Rodrigo Alberto Alandia Román, Mestre pelo programa de pós-graduação em Agronomia (Entomologia Agrícola), ambos na UNESP Campus de Jaboticabal, por auxiliarem e contribuírem para a realização desta pesquisa.

\section{Referências}

BAUER, F.C.; RAETANO, C.G. Assistência de ar na deposição e perdas de produtos fitossanitários em pulverizações na cultura da soja. Scientia Agrícola, v.57, n.2, p.271-276, 2000. 
CHAIM, A. et al. Comparison of microscopic method and computational program for pesticide deposition evaluation of spraying. Pesquisa Agropecuária Brasileira, v.37, n.4, p.493-6, 2002.

CHAIM, A. Desenvolvimento de um pulverizador eletrohidrodinâmico: avaliação do seu comportamento na produção de gotas e controle de trips (Enneothrips flavens Moulton, 1951), em amendoim (Arachis hipogaea L.). 1984. 107p. Dissertação (Mestrado em Agronomia) - Faculdade de Ciências Agronômicas e Veterinária, Universidade Estadual Paulista, Campus de Jaboticabal.

ETHERIDGE, R.E.; WOMAC, A.R.; MUELLER, T.C. Characterization of the spray droplet spectra and patterns of four venturi-type drift reduction nozzles. Weed Technology, v.13, n.4, p.765-770, 1999.

FAO. FOOD AGRICULTURE AND ORGANIZATION. Equipo de aplicación de pesticida para uso en agricultura: equipo impulsado mecánicamente. ROMA, 1997. v.2, $150 \mathrm{p}$.

FERREIRA， M.C.; OLIVEIRA， J.R.G.D.; PIETRO, I.R.P.S.D. Distribuição da calda herbicida por pontas de pulverização agrícola utilizadas em áreas de reflorestamento com eucalipto. Engenharia Agrícola, v. 29, n. 2, p. 267-276, 2009.

FOX, R.D. et al. A History of air-blast sprayer development and future prospects. American Society of Agricultural and Biological Engineers, v. 51, n. 2, p. 405-410, 2008.

LOST, C.A.R. et al. Avaliação de volumes de calda proporcionados por diferentes pontas de pulverização no controle de Tuta absoluta (Merick, 1917) (Lepidoptera: Gelechiidae) em tomate rasteiro. Acta Scientiarum Agronomy, v. 30, suplemento, p.619-624, 2008.

MAGNO JÚNIOR, R.G. Desenvolvimento de sistema eletrônico para pulverização e atração de gotas em plantas cítricas. 2008. 85f. Dissertação (Mestrado em Engenharia Agrícola) Curso de Pós-graduação em Engenharia Agrícola, Universidade Federal de Viçosa.
MATUO, T. Técnicas de aplicação de defensivos agrícolas. Jaboticabal, FUNEP, 1990, 139 p.

MATHEWS, G.A. Electrostatic spraying of pesticides: a review. Crop protection, v.8, p.3-15, 1989.

ORGANIZACIÓN MUNDIAL DE LA SALUD. Material de lucha contra los vetores. Genebra, 1976. $189 \mathrm{p}$.

PERECIN, D. et al. Padrões de distribuição obtidos com bicos TF-VS4, TJ60-11006 e TQ15006 em mesa de prova. Pesquisa Agropecuária Tropical, v.33, n.2, p.175-82, 1998.

PESSOA, M.C.P.Y., CHAIM, A. Programa computacional para estimativa de uniformidade de gotas de herbicidas aplicados por pulverização aérea. Pesquisa Agropecuária Brasileira, v.34, n.1, p.45-56, 1999.

PESSOA, M.C.P.Y.; CHAIM, A.; HERMES, L.C. Programa computacional para estimar uniformidade de gotas e volume de deposição de calda pulverizada (versão 0.5 ). Jaguariúna: EMBRAPA Meio Ambiente. 1999. 31p.

RUAS, R.A.A. et al. Desenvolvimento e avaliação de um sistema experimental de pulverização visando a determinação de parâmetros para aplicação de agrotóxicos. Engenharia Agrícola, v. 17, n.1, p. 31-41,, 2009.

SCRAMIN, S. et al. Avaliação de bicos de pulverização de agrotóxicos na cultura do algodão. Pesticidas Revista Ecotoxicologia e Meio Ambiente, v. 12, p. 43-50, 2002.

SILVA, A.R. et al. Caracterização do diâmetro de gotas de aerossóis em inseticidas domissanitários. Arquivos do Instituto Biológico, v.76, n.3, p. 437441, 2009.

SILVA, A.R.; LEITE, M.T.; FERREIRA, M.C. Estimativa da área foliar e capacidade de retenção de calda fitossanitária em cafeeiro. Bioscience Journal, v. 24, n. 3, p. 66-73, 2008.

VALE, F. X. R. et al. Quant - A software to quantify plant disease severity. In: International Workshop On Plant Disease Epidemiology; The 
International Society of Plant Pathology, 2001, Brasil, Proceedings. v.8, p. 160.

WOLF, D. D.; SMITH, E. S. Uniformity of seed and fertilizer distribution with a hand-operated spinning spreader. Transactions of the ASABE, v.22, n.4, 761-2, 1979. 